\title{
Authors Index
}

Abdel-Halim, S. T. 614

Abdel-Kader, M. H. 614

Aikawa, M. 511

Akiyama, M. 774

Aksnes, G. 435

Andolfatto, M. 651, 755

Andresen, U.. 283, 385, 469

Annis, B. K. 164

Asaad, A. N. 435

Asaji, T. 1002

Awadelkarim, O. 633

Baalss, D. 662

Baier, G. 987, 995

Baldacci, A. 331

Baluja, S. 1065

Bañón, M. L. 123

Barbero, G. 547

Barfuss, S. 758

Beavis, R. C. 1083, 1151

Beltran-Porter, D. 591

Berkemeier, J. 17

Blanco, M. C. 847

Blinc, R. 1026

Boag, N. M. 758

Boberg, L. 697

Boddenberg, B. 497

Böttcher, O. 47

Bojarski, C. 297

Borchers, D. 643

Borschel, E. M. 207

Braun, M. 291

Brener, R. 855

Brockner, W. 326, 494, 828

Brodawik, E. 823

Brosa, U. 697

Bruhn, B. 411, 930

Brummer, S. 873

Brunvoll, J. 693, 889

Buback, M. 207

Caminiti, R. 317, 591

Cerioni, G. 317

Chassot, L. 983

Chihara, H. 233

Claesson, O. 633

Cox, A. P. 271

Crisponi, G. 317

Cucca, P. 317

Cyvin, B. N. 889

Cyvin, S. J. $\quad 693,889$

Dachwitz, E. 285, 476

Das, S. P. 977

Deligeorgiev, T. 793

Dimitriou, P. 1017

Dolinšek, J. 1026

Dowben, P. A. 758

Dräger, K. 262
Dreizler, H. 133, 138 , 196, 277, 280, 283, 338, $385,469,657,755,971$

Duffner, E. 521

Eberstein, W. 755

Eckhardt, W. 314

Eichhorn, K. 391

Emrich, K. 758

Endoh, A. 638, 961, 1065

Escriña, J. A. 123

Fabian, W. 85

Fink, W. 538

Fischer, F. 1119

Fischer, K. 388, 391

Försterling, H. D. 956

Frodl, P. 914, 923

Fujii, Y. 442

Funke, K. 1094

Furukane, U. 303

Furukawa, Y. 187

Gallego, L. J. 847

Gambi, A. 331

Getoff, N. 59, 248, 430, 1126

Giesen, T. 402

Giorgianni, S. 331

Gliemann, G. 555, 983

Gonzáles-Velasco, J. 123

Gottwald, H. 481

Grassberger, P. 671

Gripp, J. 133, 971

Grotemeyer, J. 1083, 1151

Gryczyński, Z. 193, 363

Grzonka, Z. 363

Guarnieri, A. 215

Guéret, P. 684

Gutman, I. 939

Haase, W. 885

Habib, A. M. 614

Haekel, J. 203, 1111

Hahn, J. 617

Haibara, T. 1005

Hannongbua, S. V. 143, 572

Harada, K. 181

Harter, M. 402

Hau, K. 914, 923

Hawlicka, E. 769

Heineking, N. 280, 283, 657,755

Heinzinger, K. 43, 572, 1103

Herbst, B. M. 727

Herdlicka, C. 1075

Hermann, H. 177

Hermeling, J. 1094
Hess, S. 662

Heyne, U. 215

Hillig II., K. W. 271

Hirokawa, K. 187

Hirrle, R. 81

Hirschwald, W. 758

Hlavacek, V. 1017

Hofacher, G. L. 73

Horiuchi, K. 1002

Hosoe, M. 442

Igarashi, K. 289

Ikeda, R. 78, 1002

Imachi, M. 271

Inoue, A. 505, 517

Inskeep, W. H. 695

Ishida, T. 511, 572

Ishikawa, A. 1002

Iwase, M. 181

Jäger, H. 507

Jung, W. I. 291, 909

Kahlert, C. 115, 1091

Kakihana, H. 511

Kakihana, M. 774

Kakiuchi, M. 449

Kaner, I. 793

Kawski, A. 193, 297, 363

Kelly, E. M. 865

Kemmler-Sack, S. 81

Keussen, Ch. 385, 469

Khalil, S. M. 485,801 , 868

Kirfel, A. H. 388, 391

Kizler, P. 1047

Klämke, W. 885

Klages, G. 1

Kleibömer, B. 561

Klein, H. 825

Klein, M. 819, 1091

Klemm, A. 442

Kobayashi, A. 233

Koch, B. P. 930

Köster, C. 1151

Kokpol, S. U. 143

Koniński, M. 228

Krätzschmar, O. 765

Krause, H. 651

Krause, W. 509

Kreawsrikul, S. 143

Kroesen, G. M. W. 806

Kruscha, K. J. G. 93

Kruse, C. 755

Kubicki, A. 297

Kubo, A. 78

Kuczkowski, R. L. 271

Kümpers, J. 1094

Kumar, S. 1017

Kunst, M. 189

Kuśba, J. 627
LaBarge, M. S. 271

LaGraffe, D. 758

Lahajnar, G. 1026

Lakhtakia, A. 681, 943

Lakhtakia, M. N. 681

Lalowicz, Z. T. 219, 895

Lamparter, P. 1047, 1055, 1061

Landrath, K. D. 309

Lang, E. W. 538

Lehmann, G. 309, 1072

Levine, R. D. 73

Li, G. 248, 1126

Lindner, J. 1083, 1151

Lortz, D. 1009

Lund, A. 633

Lutz, O. 291, 909

Mäder, H. 203, 454, 1111

Margheritis, C. 129, 751

Maroulis, G. 419

Massen, S. E. 821

Masumoto, T. 505, 517

Matsubara, E. 181,505

Mattern, N. 177

Matz, W. 177

Mauer, D. 617

Mehrotra, S. C. 454

Meidenbauer, K. 555

Meier, H. 583

Mellander, B. E. 1044

Merke, I. 196

Messier, R. 943

Metzler, W. 14, 287

Meyer, M. 138

Milia, F. 1026

Minchev, St. 59

Miraldi, E. 547

Misawa, M. 961

Mochinaga, J. 289

Montjoie, A.-S. 35

Moroishi, K. 517

Müller, B. 1007

Müller-Buschbaum, H. K. 965

Müller-Warmuth, W. 35, 219

Nagumo, T. 774

Nakamura, D. 78, 233, 1002

Nalewajski, R. F. 65,228

Narten, A. H. 164

Neger, T. 507

Neue, G. 30

Nikolov, P. 59, 793

Nomura, M. 91, 1005

Novak, I. 851

Oda, T. 303

Odawara, O. 961, 1005 
Oehler, S. $\quad 687,828$

Oelkrug, D. 583

Oeschey, R. 291

Ohse, L. 326, 494, 828

Ohtaki, H. 961

Oi, T. 511

Okada, I. 91, 638, 961, 1005,1065

Okamoto, M. 91, 774, 1005

Oldano, C. 547

Ottinger, Ch. 1007

Pálinkás, G. 43

Papadopoulos, M. G. 253

Park, H. R. 430, 1126

Parisi, J. 14, 287

Paulus, H. 873

Peinke, J. 14, 287

Petcov, A. 388

Pfirsch, D. 395, 521

Pintar, M. M. 1026

Pirzer, G. 983

Pohl, F. 395

Polacki, Z. 621

Polman, S. 143

Pompe, B. 93

Prass, E. 583

Preetz, W. 239

Purwins, H.-G. 17

Puszynski, J. 1017

Quixiang, W. 693

Radehaus, C. 17

Radkowitsch, H. 538

Randell, J. 271

Rak, J. 621

Reinartz, K. 617
Rempfer, K. 583

Richter, J. 1075

Rieckers, A. 521

Roca, C. M. 591

Rode, B. M. 143, 797

Röhricht, B. 14, 287

Rössler, O. E. 14, 110, 287

Rohwer, E. R. 1151

Rossi, A. 591

Roy, P. 360

Roychoudhoury, R. 360

Ruangpornvisuti, V. W. 797

Salem, M. A. 614

Sallhofer, H. 1039

Sartori, C. 239

Sastry, S. S. 741

Satyanandam, G. 741

Schaal, M. 1055, 1061

Schieder, R. 402

Schindler, K.-M. 189

Schlag, E. W. 765, 1083 , 1151

Schmidt, P. C. 643

Schmiedel, H. $\quad 687,828$

Schmitz, G. 825

Schram, D. C. 806

Schreiber, H. 956

Schwarz, R. 277

Selzle, H. L. 765

Sepe, A. 1026

Shechter, H. 855

Shimada, M. 289

Sinistri, C. 129,751

Somer, M. 326, 828

Sommer, F. 914, 923

Somoza, J. A. 847
Spohr, E. 43, 572, 1103

Stahl, W. 280

Stanislawski, J. 35

Stannarius, R. $\quad 687,828$

Stauf, G. T. 758

Steeb, S. 1047, 1055, 1061

Steeb, W.-H. 727

Stevens, J. R. 1044

Stiefvater, O. L. 147, 155, 597,607

Stiller, H. 35

Stockhausen, M. 476

Stumpf, H. 345

Sugiyama, K. 517

Sundar Raj, T. F. 741

Sutter, D. H. 47, 561, 651

Szyncel, M. 105

Tai, Y. 1002

Tamura, Y. 43, 961, 1103

Tanaka, K. 1103

Tél, T. 1154

Tempczyk, A. 363, 621

Teske, C. L. 865

Thiem, J. 1119

Timmermans, C. J. 806

Timtcheva, I. 59

Tsai, A. P. 505

Tsuji, Y. 303

Twardowski, R. 627

Tyutyulkov, N. 793

Urban, P. 995, 987

Valabrega, P. T. 547

Vallinga, P. M. 806

Varadan, V. K. 943

Varadan, V. V. 943

Vassilikou-Dova, A. B. 309, 1072
Venkata Rao, V. L. 741

Vill, V. 1119

Vormann, K. 277, 283 338

Wahl, F. 914, 923

Waite, J. 253

Wallschläger, H. 829

Wang, S. 248

Waseda, Y. 505, 517

Wegmann, K. 987,995

Weil, K. G. 481, 977

Weiss, Al. 643, 873

Wenchen, H. 693

Wenjie, H. 693

Werner, U. 219

Wilhelm, H. E. 859

Winnewisser, G. 402, 407 , 617

Winterberg, F. 169, 369, 1151

Winther, F. 215

Witko, M. 823

Wittekopf, B. 977

Yamada, K. M. T. 407, 617

Yamaguchi, T. 961

Yang, Ch. 91

Yoshioka, Y. 233

Yukhnovskii, I. R. 734

Yurtsever, E. 745

Zachara, S. 105

Zander, M. 393

Zechner, J. 59

Zeidler, M. D. 1075

Zelewsky, A. von 983

Žumer, S. 1026

Zupančič, I. 1026

Zurkowska, G. 297 